\title{
Digital Phase Reconstruction via Iterative Solutions of Transport-of-Intensity Equation
}

\author{
Emmanuel Froustey, Emrah Bostan, Stamatios Lefkimmiatis and Michael Unser \\ Biomedical Imaging Group, EPFL, Lausanne, Switzerland
}

\begin{abstract}
We develop a variational algorithm for reconstructing phase objects from a series of bright field micrographs. Our mathematical model is based on the transport-of-intensity equation (TIE), which links the phase of a complex field to the axial derivative of its intensity. To reduce reconstruction artifacts, we formulate TIE in a regularized fashion by introducing a family of penalty functionals based on the eigenvalues of the structure tensor. To solve the arising optimization problem, we propose an algorithm based on the alternating direction method of multipliers (ADMM). We apply our method on simulated data and illustrate improved performance compared to the conventional methods such as Tikhonov and total variation (TV) regularizations. We further demonstrate the applicability of the proposed approach by applying it to experimentally-acquired bright field data.

Keywords-Phase imaging; transport-of-intensity; variational method; structure tensor total variation regularization.
\end{abstract}

\section{INTRODUCTION}

Many biological specimens, such as cells and organisms, are transparent or weakly absorbant. In the absence of any staining, they remain almost invisible under a conventional bright field microscope. However, their variable thickness and refractive index induce differences of optical path length in the light wave that illuminates them. Important biological information are thus encoded in the phase, which makes phase retrieval a fundamental problem in biology, for which many techniques have been developed [1].

In this work we consider a digital phase imaging approach relying on the transport-of-intensity equation (TIE) [2]. Under the paraxial approximation, TIE states that the phase information is contained in the variations of the intensity along the propagation. Mathematically, this relation is stated in the form of a linear second-order partial differential equation. Thus, by acquiring several through-focus intensity images, one is able to reconstruct the phase map, which makes of TIE a conveniently purely computational method.

A classical approach to reconstruct phase maps with TIE is to solve the equation directly via a Fourier transform [3]. However, this procedure integrates the noise present in the measurements, which results in "cloudy", low-frequency artifacts. To reduce this phenomenon, regularized solutions have been proposed, including Tikhonov [4], total variation (TV) and nonlinear diffusion regularization schemes [5]. We propose here a regularization strategy that further improves the reconstruction quality.

This work was supported by the European Research Council under Grant ERC-2010-AdG 267439-FUN-SP
The main contributions of this work are as follows:

- The use of a recently introduced family of penalty functionals based on the eigenvalues of the structure tensor for solving TIE. These regularizers are able to measure the variations at every pixel by taking into account the information from its neighborhood. This family generalizes several existing variational penalties, including TV. In this regard, the proposed framework provides a rich class of regularized solutions of the TIE- based phase reconstruction.

- An iterative reconstruction algorithm based on the alternating direction method of multipliers (ADMM), to solve the corresponding optimization problem. This approach decomposes the minimization at hand into smaller problems that are solved efficiently.

- The application of our method on simulated data, where we make comparisons of different solutions of TIE. In particular, we show numerical improvements compared to Tikhonov and TV regularization. We also apply our method to real bright field images of HeLa cells.

The remainder of this work is organised as follows: in Section II, we introduce our forward model, give the mathematical detail of our regularisation method, and present our algorithm. In Section III, we perform experiments on simulated data and show a real data reconstruction.

\section{MODEL}

\section{A. Physical model}

Consider a monochromatic scalar field propagating dominantly in the $+z$-direction. In a tranverse plane $z \in \mathbb{R}$ and over a bounded domain indexed by the coordinate $\mathbf{r}=(x, y) \in \mathbb{R}^{2}$, the complex amplitude of the wave can be written

$$
U(\mathbf{r}, z)=\sqrt{I(\mathbf{r}, z)} \mathrm{e}^{j \phi(\mathbf{r}, z)},
$$

where $I$ and $\phi$ denote the intensity and phase, respectively. During a classical acquisition, one measures the instensity $I=$ $|U|^{2}$, and the phase information is lost. To circumvent this issue, Teague derived the transport-of-intensity equation (TIE) in [2]. Under the assumption of a phase-only object, it reads

$$
-\frac{k}{I(\mathbf{r}, z)} \frac{\partial}{\partial z} I(\mathbf{r}, z)=\nabla_{\perp}^{2} \phi(\mathbf{r}, z),
$$

where $k$ is the wavenumber and $\nabla_{\perp}^{2}$ denotes the Laplacian operator in the transverse plane. 
We compute the left hand side of (1) by acquiring three through-focus intensity images, and applying a centered finite difference formula.

\section{B. Inverse problem formulation}

Let us now consider the following discretized forward model:

$$
\mathbf{y}=\mathbf{H} \phi+\mathbf{n},
$$

where $\mathbf{y} \in \mathbb{R}^{N}$ is the measurement computed as described above, $\mathbf{H} \in \mathbb{R}^{N \times N}$ is a matrix representation of the discrete Laplacian, $\phi \in \mathbb{R}^{N}$ is the unknown phase and $\mathbf{n} \in \mathbb{R}^{N}$ is an additive noise contribution. We assume that the images contain $N$ pixels and have been reordered into single-column vectors.

In order to cure the ill-posedness introduced by the noise contribution, we formulate (2) as an energy minimization problem

$$
\hat{\boldsymbol{\phi}}=\min _{\boldsymbol{\phi}} \frac{1}{2}\|\mathbf{H} \boldsymbol{\phi}-\mathbf{y}\|_{2}^{2}+\tau R(\boldsymbol{\phi}),
$$

where $\tau>0$ is a tuning parameter and $R(\cdot)$ is a regularization functional (also called the penalty functional). For its ability to produce a flat background and preserve sharp edges in the reconstruction, we choose to use a regularizer based on the eigenvalues of the structure tensor [6]. In the sequel, we provide further information on our regularizers.

Let $\mathbf{K} \in \mathbb{R}^{L}$ be a discrete, non-negative, rotationally symmetric convolution kernel defined on the finite lattice $\mathcal{S}=\left\{-L_{\mathbf{K}}, \ldots, L_{\mathbf{K}}\right\}^{2}$, with $L_{\mathbf{K}} \in \mathbb{N}$ and $L=\left(2 L_{\mathbf{K}}+1\right)^{2}(\mathbf{K}$ may be for example a truncated Gaussian). The patch-based Jacobian of $\phi$ is defined at each pixel $i \in\{1, \ldots, N\}$ by

$$
\left[\mathbf{J}_{\mathbf{K}} \boldsymbol{\phi}\right]_{i}=\left[\begin{array}{cc}
{\left[\mathbf{P}_{\mathbf{s}_{1}} \mathbf{D}_{h} \boldsymbol{\phi}\right]_{i}} & {\left[\mathbf{P}_{\mathbf{s}_{1}} \mathbf{D}_{v} \boldsymbol{\phi}\right]_{i}} \\
\vdots & \vdots \\
{\left[\mathbf{P}_{\mathbf{s}_{L}} \mathbf{D}_{h} \boldsymbol{\phi}\right]_{i}} & {\left[\mathbf{P}_{\mathbf{s}_{L}} \mathbf{D}_{v} \boldsymbol{\phi}\right]_{i}}
\end{array}\right],
$$

where $\mathbf{D}_{h}$ and $\mathbf{D}_{v}$ are the horizontal and vertical components of the discrete gradient, the shift vectors $\mathbf{s}_{l}$ belong to the lattice $\mathcal{S}$ with $l \in\{1, \ldots, L\}$, and the weighted shift operators $\mathbf{P}_{\mathbf{s}_{l}}$ are defined as

$$
\left[\mathbf{P}_{\mathbf{s}_{l}} \mathbf{D}_{h} \phi\right]_{i}=\mathbf{K}\left[\mathbf{s}_{l}\right] \mathbf{D}_{h}\{\boldsymbol{\phi}\}\left[\mathbf{r}_{i}-\mathbf{s}_{l}\right] .
$$

Having introduced the patch-based Jacobian, the structure tensor is defined at each pixel by

$$
\left[\mathbf{S}_{\mathbf{K}} \boldsymbol{\phi}\right]_{i}=\left[\mathbf{J}_{\mathbf{K}} \phi\right]_{i}^{T}\left[\mathbf{J}_{\mathbf{K}} \boldsymbol{\phi}\right]_{i} .
$$

If we denote by $\lambda_{1, i}$ and $\lambda_{2, i}$ the eigenvalues of $\left[\mathbf{S}_{\mathbf{K}} \phi\right]_{i}$, the associated penalty functionals for the regularization are

$$
R(\phi)=\sum_{i=1}^{N}\left\|\left(\sqrt{\lambda_{1, i}}, \sqrt{\lambda_{2, i}}\right)\right\|_{p}
$$

where $p \geq 1$. As shown in [6], (4) can be rewritten

$$
R(\phi)=\sum_{i=1}^{N}\left\|\left[\mathbf{J}_{\mathbf{K}} \phi\right]_{i}\right\|_{\mathcal{S}_{p}}
$$

where the Schatten-norm $\|\cdot\|_{\mathcal{S}_{p}}$ of a matrix is defined as the $\ell_{p}$-norm of the vector of its singular values. This last equation allows for a more practical computation of $R(\phi)$ since the patch-based Jacobian, unlike the structure tensor, is a linear operator of $\phi$.

The choices of $\mathbf{K}$ and $p$ generate a whole family of functionals. In our experiments we shall consider $\mathbf{K}$ to be a Gaussian kernel, and $p \in\{1,2, \infty\}$. Following the notations of [6], we denote the three corresponding models by STV$\mathrm{N}$ (Structure tensor Total Variation-Nuclear norm), STV-F (Frobenius norm), and STV-S (Spectral norm), respectively. For comparison purposes, we shall also consider the case when $\mathbf{K}$ is the Dirac's delta function, which reduces the regularisation to the classical TV scheme.

\section{Algorithm}

We choose to solve (3) by the alternating direction method of multipliers (ADMM) [7], for its ability to decompose the optimization problem at hand into smaller sub-problems each of which can be solved efficiently. The first step is to rewrite (3) in a constrained form, introducing the auxiliary variable $\mathbf{z}$ :

$$
\hat{\boldsymbol{\phi}}=\min _{\boldsymbol{\phi}} \frac{1}{2}\|\mathbf{H} \boldsymbol{\phi}-\mathbf{y}\|_{2}^{2}+\tau \sum_{i=1}^{N}\left\|\mathbf{z}_{i}\right\|_{\mathcal{S}_{p}}, \quad: \quad \mathbf{z}=\mathbf{J}_{\mathbf{K}} \boldsymbol{\phi} .
$$

Then we introduce the augmented Lagrangian of (5) with the dual variable $\alpha$ and the penalty parameter $\mu>0$

$$
\begin{aligned}
\mathcal{L}(\boldsymbol{\phi}, \mathbf{z} ; \boldsymbol{\alpha}) & =\frac{1}{2}\|\mathbf{H} \boldsymbol{\phi}-\mathbf{y}\|_{2}^{2}+\tau \sum_{i=1}^{N}\left\|\mathbf{z}_{i}\right\|_{\mathcal{S}_{p}} \\
& +\left\langle\mathbf{z}-\mathbf{J}_{\mathbf{K}} \boldsymbol{\phi}, \boldsymbol{\alpha}\right\rangle_{\mathcal{X}}+\frac{\mu}{2}\left\|\mathbf{z}-\mathbf{J}_{\mathbf{K}} \boldsymbol{\phi}\right\|_{\mathcal{X}}^{2},
\end{aligned}
$$

where we denote the space of matrix fields by $\mathcal{X}$ for which the inner product and norm are defined by $\langle\mathbf{X}, \mathbf{Y}\rangle_{\mathcal{X}}=$ $\sum_{i=1}^{N} \operatorname{tr}\left(\mathbf{Y}_{i}^{T} \mathbf{X}_{i}\right)$ and $\|\mathbf{X}\|_{\mathcal{X}}=\sqrt{\langle\mathbf{X}, \mathbf{X}\rangle_{\mathcal{X}}}$, respectively. To find the saddle point of (6), ADMM updates iteratively the variables according to the following algorithm:

$$
\begin{aligned}
\mathbf{z}^{t+1} & \leftarrow \underset{\mathbf{z}}{\operatorname{argmin}} \mathcal{L}\left(\boldsymbol{\phi}^{t}, \mathbf{z} ; \boldsymbol{\alpha}^{t}\right), \\
\boldsymbol{\phi}^{t+1} & \leftarrow \underset{\boldsymbol{\phi}}{\operatorname{argmin}} \mathcal{L}\left(\boldsymbol{\phi}, \mathbf{z}^{t+1} ; \boldsymbol{\alpha}^{t}\right), \\
\boldsymbol{\alpha}^{t+1} & \leftarrow \boldsymbol{\alpha}^{t}+\mu\left(\mathbf{z}^{t+1}-\mathbf{J}_{\mathbf{K}} \boldsymbol{\phi}^{t+1}\right) .
\end{aligned}
$$

We refer the reader to [6] for the details regarding the implementation of (7). Equation (8) is a quadratic minimization whose solution is given by

$$
\phi^{t+1}=\left(\mathbf{H}^{T} \mathbf{H}+\mu \mathbf{J}_{\mathbf{K}}^{T} \mathbf{J}_{\mathbf{K}}\right)^{-1}\left(\mathbf{H y}+\mu \mathbf{J}_{\mathbf{K}}^{T}\left(\mathbf{z}^{t+1}-\frac{\boldsymbol{\alpha}^{t}}{\mu}\right)\right),
$$

and can be implemented in one step by FFT. Finally (9) is a trivial gradient ascent.

\section{EXPERIMENTAL RESULTS}

\section{A. Simulated data}

We simulate the measurement of three through-focus intensity images by propagating a complex field with a Fresnel diffraction kernel [2], [8]. Assuming phase-only objects, we set the infocus intensity to one and use 3 different phase maps 

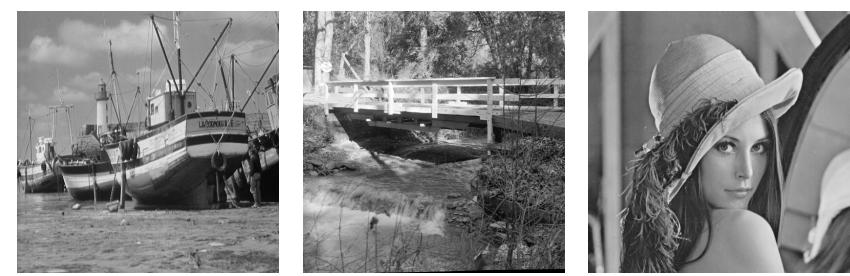

Fig. 1. Phase maps used for the simulations: Boat, Bridge and Lena.

TABLE I

COMPARISON OF RECONSTRUCTION SNR FOR THREE NOISE LEVELS

\begin{tabular}{|c|c|c|c|c|c|c|c|}
\hline \multicolumn{2}{|c|}{ Input SNR } & Direct & Tikh. & TV & STV-N & STV-F & STV-S \\
\hline \multirow{3}{*}{$\underset{8}{\tilde{\Xi}}$} & 0 & 10.78 & 10.91 & 12.09 & 12.21 & 12.06 & 12.03 \\
\hline & 10 & 10.47 & 13.09 & 15.61 & 15.80 & 15.51 & 15.38 \\
\hline & 20 & 13.99 & 14.24 & 18.41 & 18.63 & 18.33 & 18.23 \\
\hline \multirow{3}{*}{ ס } & 0 & 8.47 & 8.66 & 9.39 & 9.46 & 9.38 & 9.38 \\
\hline & 10 & 7.85 & 9.98 & 11.76 & 11.91 & 11.74 & 11.68 \\
\hline & 20 & 10.97 & 11.85 & 14.32 & 14.38 & 14.30 & 14.27 \\
\hline \multirow{3}{*}{ క్ర } & 0 & 9.29 & 10.89 & 12.73 & 12.85 & 12.76 & 12.77 \\
\hline & 10 & 9.53 & 13.18 & 16.47 & 16.62 & 16.54 & 16.53 \\
\hline & 20 & 16.81 & 19.26 & 20.28 & 20.31 & 20.35 & 20.34 \\
\hline
\end{tabular}

shown in Figure 1. We degrade each set of images by adding a white Gaussian noise, in order to reach three different input signal-to-noise ratios (SNR).

For each algorithm, the parameter $\tau$ is optimized for highest-possible output SNR, and we set $\mu=10 \tau$. We perform 500 iterations of ADMM, which is observed to be enough for convergence. The reconstruction SNRs are given in Table I for direct reconstruction, Tikhonov regularization, TV, and the three STV methods mentioned previously. We observe that the structure tensor-based regularization achieve better reconstruction quality, in particular with a significant improvement over the direct and Tikhonov reconstructions.

\section{B. Real data}

We illustrate the applicability of our method on real data by reconstructing the phase map of a HeLa cell culture. The images were acquired on a Zeiss Axio Observer Z1 (Carl Zeiss AG, Jena, Germany) equipped with an APlan $10 \times / 0.25$ NA. The defocus distance was $2 \mu \mathrm{m}$ from the best focus position.

Figure 2 shows that in comparison with direct and Tikhonov reconstructions, TVS-N produces the phase map with the flattest background, while preserving the details of the cells shapes and structures.

\section{CONCLUSION}

We solved the TIE in a variational framework, using a family of regularizers based on the eigenvalues of the structure tensor. Proposing an iterative algorithm, we showed numerical improvement over Tikhonov and TV regularizations. Finally, we demonstrated that our method can be used for real data reconstructions.

\section{ACKNOWLEDGMENT}

The authors would like to thank Benjamin Rappaz from the Biomolecular Screening Facility, EPFL, and Etienne Shaffer

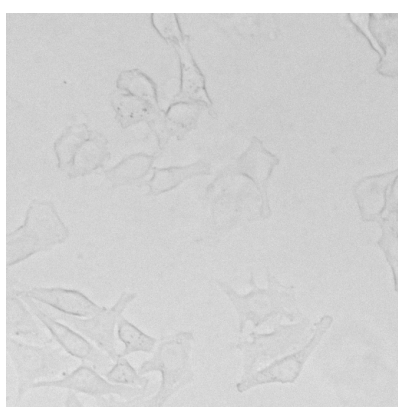

(a)

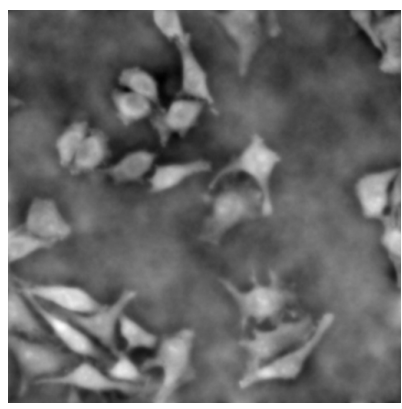

(c)

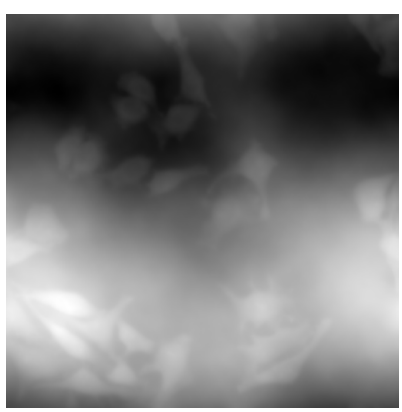

(b)

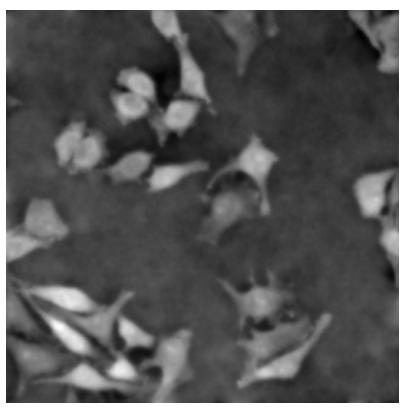

(d)
Fig. 2. HeLa cells (cropped). Comparison of TIE reconstructions. (a) Bright field in-focus image, (b) Direct reconstruction, (c) Tikhonov, (d) STV-N.

from the Microsystems Laboratory 1, EPFL, for their help in acquiring the experimental data.

\section{REFERENCES}

[1] G. Popescu, Quantitative phase imaging of cells and tissues. McGraw Hill Professional, 2011.

[2] M. R. Teague, "Deterministic phase retrieval: a green's function solution," Journal of the Optical Society of America, vol. 73, no. 11, pp. 1434-1441, 1983.

[3] T. E. Gureyev and K. A. Nugent, "Rapid quantitative phase imaging using the transport of intensity equation," Optics Communications, vol. 133, no. 1, pp. 339-346, 1997.

[4] M. Mitome, K. Ishizuka, and Y. Bando, "Quantitativeness of phase measurement by transport of intensity equation," Journal of electron microscopy, vol. 59, no. 1, pp. 33-41, 2010.

[5] L. Tian, J. C. Petruccelli, and G. Barbastathis, "Nonlinear diffusion regularization for transport of intensity phase imaging," Optics Letters, vol. 37, no. 19, pp. 4131-4133, 2012.

[6] S. Lefkimmiatis, A. Roussos, M. Unser, and P. Maragos, "Convex generalizations of total variation based on the structure tensor with applications to inverse problems," in Proceedings of the Fourth International Conference on Scale Space and Variational Methods in Computer Vision (SSVM'13), 2013.

[7] S. Boyd, N. Parikh, E. Chu, B. Peleato, and J. Eckstein, "Distributed optimization and statistical learning via the alternating direction method of multipliers," Foundations and Trends $\AA$ in Machine Learning, vol. 3, no. 1, pp. 1-122, 2011.

[8] M. Liebling, T. Blu, and M. Unser, "Fresnelets: New multiresolution wavelet bases for digital holography," IEEE Transactions on Image Processing, vol. 12, no. 1, pp. 29-43, 2003. 